\title{
UCRL-PROC-226945
}

LAW RENCE LIVERMORE N A TIO N A L LABORATORY

\section{The Injection Laser System on the National Ignition Facility}

M. Bowers, S. Burkhart, S. Cohen, G. Erbert, J. Heebner, M. Hermann, D. Jedlovec

December 21, 2006

SPIE Photonics West

San Jose, CA, United States

January 20, 2007 through January 25, 2007 
This document was prepared as an account of work sponsored by an agency of the United States Government. Neither the United States Government nor the University of California nor any of their employees, makes any warranty, express or implied, or assumes any legal liability or responsibility for the accuracy, completeness, or usefulness of any information, apparatus, product, or process disclosed, or represents that its use would not infringe privately owned rights. Reference herein to any specific commercial product, process, or service by trade name, trademark, manufacturer, or otherwise, does not necessarily constitute or imply its endorsement, recommendation, or favoring by the United States Government or the University of California. The views and opinions of authors expressed herein do not necessarily state or reflect those of the United States Government or the University of California, and shall not be used for advertising or product endorsement purposes. 


\title{
The Injection Laser System on the National Ignition Facility
}

\author{
Mark Bowers*, Scott Burkhart, Simon Cohen, \\ Gaylen Erbert, John Heebner, Mark Hermann, Don Jedlovec \\ Lawrence Livermore National Laboratory, 7000 East Ave, Livermore, CA 94550
}

\begin{abstract}
The National Ignition Facility (NIF) is currently the largest and most energetic laser system in the world. The main amplifiers are driven by the Injection Laser System comprised of the master oscillators, optical preamplifiers, temporal pulse shaping and spatial beam formatting elements and injection diagnostics. Starting with two fiber oscillators separated by up to a few angstroms, the pulse is phase modulated to suppress SBS and enhance spatial smoothing, amplified, split into 48 individual fibers, and then temporally shaped by an arbitrary waveform generator. Residual amplitude modulation induced in the preamplifiers from the phase modulation is also precompensated in the fiber portion of the system before it is injected into the 48 pre-amplifier modules (PAMs). Each of the PAMs amplifies the light from the $1 \mathrm{~nJ}$ fiber injection up to the multi-joule level in two stages. Between the two stages the pre-pulse is suppressed by $60 \mathrm{~dB}$ and the beam is spatially formatted to a square aperture with precompensation for the nonuniform gain profile of the main laser. The input sensor package is used to align the output of each PAM to the main laser and acquire energy, power, and spatial profiles for all shots. The beam transport sections split the beam from each PAM into four main laser beams (with optical isolation) forming the 192 beams of the NIF. Optical, electrical, and mechanical design considerations for long term reliability and availability will be discussed. Work performed under the auspices of the U. S. Department of Energy under contract W-7405-Eng-48.
\end{abstract}

Keywords: Lasers, High Energy Lasers, Solid State Lasers, Fiber Lasers

\section{INTRODUCTION}

The National Ignition Facility ${ }^{1,2}$ under construction in Livermore, California will deliver a total $1.8 \mathrm{MJ}$ at $351 \mathrm{~nm}$ when complete. The light originates in the Injection Laser system ${ }^{3,4}$ (ILS) which is composed of the master oscillator (MOR), the Pre-A mplifier Module ${ }^{5,6}$ (PAM), the Input Sensor Package (ISP) and the Pre-Amplifier Beam Transport System (PABTS). In this configuration, there are two master oscillators which each generate a $960 \mathrm{~Hz}$ optical train of pulses. Each pulse must reliably have $3 \mathrm{GHz}$ modulation with a modulation index of 5.5 in order to assure that the main laser does not initiate stimulated Brillouin scattering (SBS) which could damage the main laser optical components ${ }^{7}$. A failsafe system is used to monitor the SBS bandwidth and prevent the propagation of any pulse with less than the required modulation index. After phase modulation, the beams are amplified and split into 48 individual beams. The temporal shape of each of the 48 beams is controlled in the amplitude modulation chassis. Since the fluence in the main laser can be more than 5 times higher than the saturation fluence of the slab amplifiers it is necessary that the pulse shape be precisely controlled in a reliable manner to prevent damage in the main laser. Often the pulse shape requirements for various experimental campaigns are even more stringent than the damage restrictions in the main laser. For these reasons, the temporal shape control in the Amplitude Modulation Chassis must be tightly controlled and monitored.

The Regenerative Amplifiers (Regens) in each of the 48 PAMs are designed to operate at $1 \mathrm{~Hz}$ in a highly stable regime for energy, spatial pulse shape, temporal shape, and optical pre-pulse. The design of the Regen is such that even the slightest pulse energy fluctuations around the optimal $750 \mathrm{pJ}$ levelfrom the master oscillator are reduced to less than $1 \%$ at the $20 \mathrm{~mJ}$ Regen output ${ }^{8,9}$. Following the Regen, the beam passes through a Pockels cell pulse slicer to reduce the pre-pulse by an additional 3 orders of magnitude before passing through the Beam Shaping Module. The ISP is used to align the output of the Regen to the Beam Shaping Mask such that the maximum deviation from the optimal shape is less than 10\%. The beam then transmits through the Multi-Pass Amplifier (MPA, 4-pass) system in each PAM where it is amplified up to 6 joules. The MPA is designed to minimize the possibility of parasitics (unwanted oscillations) and pencil beams (undesired amplification of reflections from transmitting

\footnotetext{
* *bowers5@1lnl.gov; phone 1925423 0901; fax 1925422 9528; www.llnl.gov
} 
surfaces). The PAM output is aligned to the $\mathrm{cw}$ beam from the ISP in order to allow the parallel operations of setting the Regen ouput energy and aligning the main laser during a system shot cycle. This necessitates that both the PAM output and the ISP output have highly stable beam pointing characteristics and excellent beam quality.

The ISP contains the pointing and centering references used to align the PAM and establish alignment into the main laser system. In addition, it also contains the diagnostics required to assure that the correct temporal shape, spatial shape, and energy will be injected into the main laser. It is required that these diagnostics be highly repeatable and reliable, while providing good absolute accuracy. Included in the ISP is a back reflection sensor and isolation package. This isolation prevents any undesired light from the main laser from entering into the PAM potentially causing catastrophic damage. Every system shot is monitored for changes in the backreflected energy for early signs of possible problems in the optical components of the main amplifiers.

Following the ISP the beam is split using polarization splitters and adjustable waveplates to set the energy independently in each of four beams. The four-way splitter was designed to account for the less than $1 \%$ leakage of the undesired polarization which can affect the energy accuracy on target. The beam size in the PABTS area was chosen to optimize the size of the beam on each optic allowing for the diffraction effects from the grating (beam smoothing by spectral dispersion) in the PAM. Since the beam propagates more than 18 meters in the PABTS area with as many as 10 mirrors in a beamline without adjustment, the optical mounts were designed and tested to have high long term stability.

Details of how these concepts were accomplished will be presented and are described in more detail in the text below.

\section{THE MASTER OSCILLATOR ROOM}

\subsection{Introduction}

The Master Oscillator Room (MOR) of the National Ignition facility contains an all fiber based laser system used to generate the initial pulse that seeds the entire NIF laser system. The laser puls e is phase modulated to add bandwidth, and then amplified and split into 48 separate beam lines all in single-mode polarizing fiber. In addition to phase modulation, the pulse is also amplitude modulated to compensate for FM-to-AM effects throughout the NIF laser system. Before leaving the MOR, each of the 48 output beams are temporally sculpted into high contrast shapes using Arbitrary Waveform Generators. The all fiber based system implements highly reliable industrial telecom hardware. Special polarizing fiber (PZ) used in the MOR was specifically developed for use in the NIF laser system. Unlike polarization maintaining (PM) fiber, PZ fiber polarizes the light by having high loss for the fast axis component. This feature is critical in minimizing FM-to-AM effects caused by polarization mixing observed in PM fibers. The major functions of the MOR are shown in the block diagram in Figure 1.

\subsection{MOR Oscillator}

The 1.8 Mega-joules of energy at the output of the NIF laser system starts with a few mW fro m two separate fiber oscillators in the MOR. The two oscillators generate two distinct wavelengths a few angstroms apart as required by

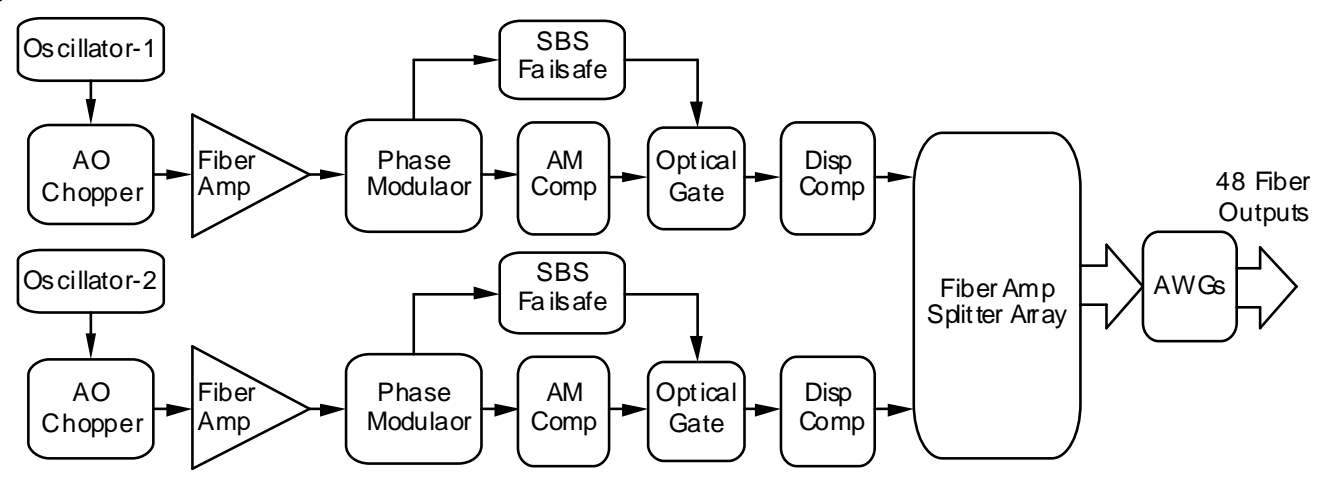

Figure 1. Functional block diagram of the MOR 
the NIF laser system. The oscillator design is a distributed feedback (DFB) laser made by imprinting linear Bragg gratings in a 2.8-micron core rare earth Ytterbium doped Silica fiber ${ }^{10}$. The Bragg gratings form distributed reflectors that define the laser cavity and allow only a single cavity mode to lase. The oscillator produces up to $20 \mathrm{~mW}$ of $\mathrm{CW}$ power in a single longitudinal mode when pumped with $130 \mathrm{~mW}$ from a $980 \mathrm{~nm}$ laser diode. The master oscillator wavelength is precisely tuned to within $0.01 \mathrm{~nm}$ of the desired value of $1053.01 \mathrm{~nm}$ by temperaturecontrolling its mount.

The performance of the oscillator is verified with a series of tests prior to integration in the main operating system. Tests include wavelength and power stability, signal to noise, and single-mode operation. Figure 2. displays the wavelength of the oscillator over a 3 -month period. The wavelength stability exceeds the NIF specification of $\pm 0.01 \mathrm{~nm}$ by almost an order of magnitude. The DFB fiber oscillator design has proven to be a reliable and stable source for NIF.

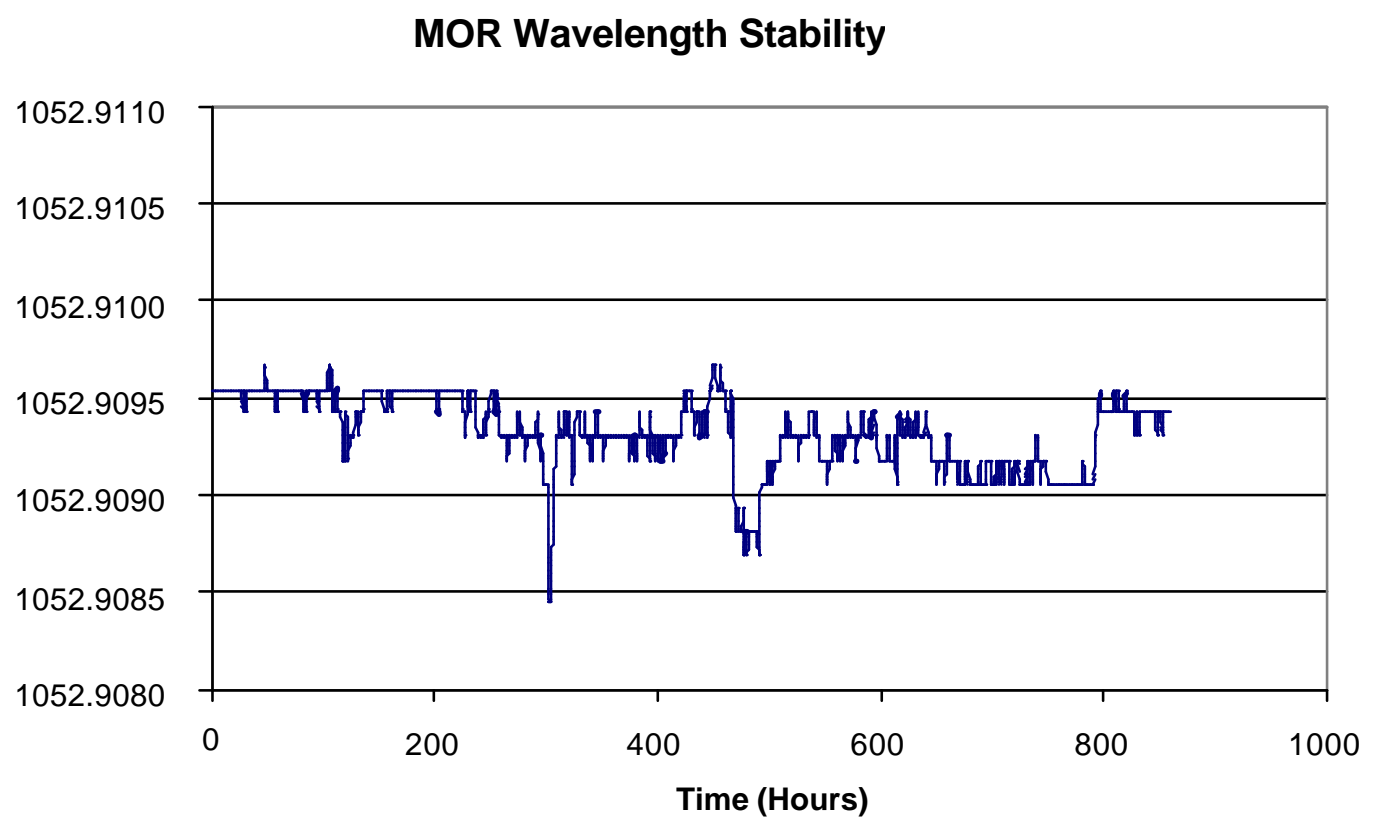

Figure 2. Oscillator wavelength as a function of time over a 3 month period.

\subsection{AO Modulator}

The CW signal from the output of the oscillator is chopped by an acousto-optic (AO) modulator to a pulse width of $100 \mathrm{~ns}$ at a pulse repetition rate of $960 \mathrm{~Hz}$. The custom AO design utilizes a commercial bulk Tellurium Dio xide $\left(\mathrm{TeO}_{2}\right)$ modulator integrated in a fiber-to-fiber micro-bench package. The $\mathrm{AO}$ modulator operates in first order at $\sim 85 \%$ diffraction efficiency. The total insertion loss is less than $2.5 \mathrm{~dB}$. The AO modulator provides $50 \mathrm{~dB}$ of isolation between the fiber oscillator and the first stage of amplification.

\subsection{Fiber Amplifiers}

The output of the AO Chopper is amplified in a series of fiber amplifiers. The amplifiers developed at LLNL consist of two stages of $\mathrm{Yb}$-doped fiber with a 2.8 micron core diameter. The fiber is $\mathrm{CW}$ pumped with a $200 \mathrm{~mW}, 980 \mathrm{~nm}$ laser diode. An optical schematic of the amplifier is shown in Figure 3. An optical filter is used between the two gain stages to prevent saturation of the amplifier by ASE. The optical filter transmits the pump and signal light to the second gain stage but rejects the strong ASE signal centered at $1020 \mathrm{~nm}$. The same optical filter is also used in front of the first gain stage to prevent backward propagating ASE from entering the pump leg of the circuit and possibly damaging the pump diode. This filter also helps to reduce any ASE from the preceding amplifier. All of the components in the fiber amplifiers use single mode fiber so the input polarization to the amplifier is not necessarily maintained at the output. A commercial polarization controller is used at the output of the amplifier to correct the 
polarization. The port-to-port small signal gain of the fiber amplifier is greater than $27 \mathrm{~dB}$ and is capable of generating over 1.5 micro-joules of energy in a 45 ns pulse.

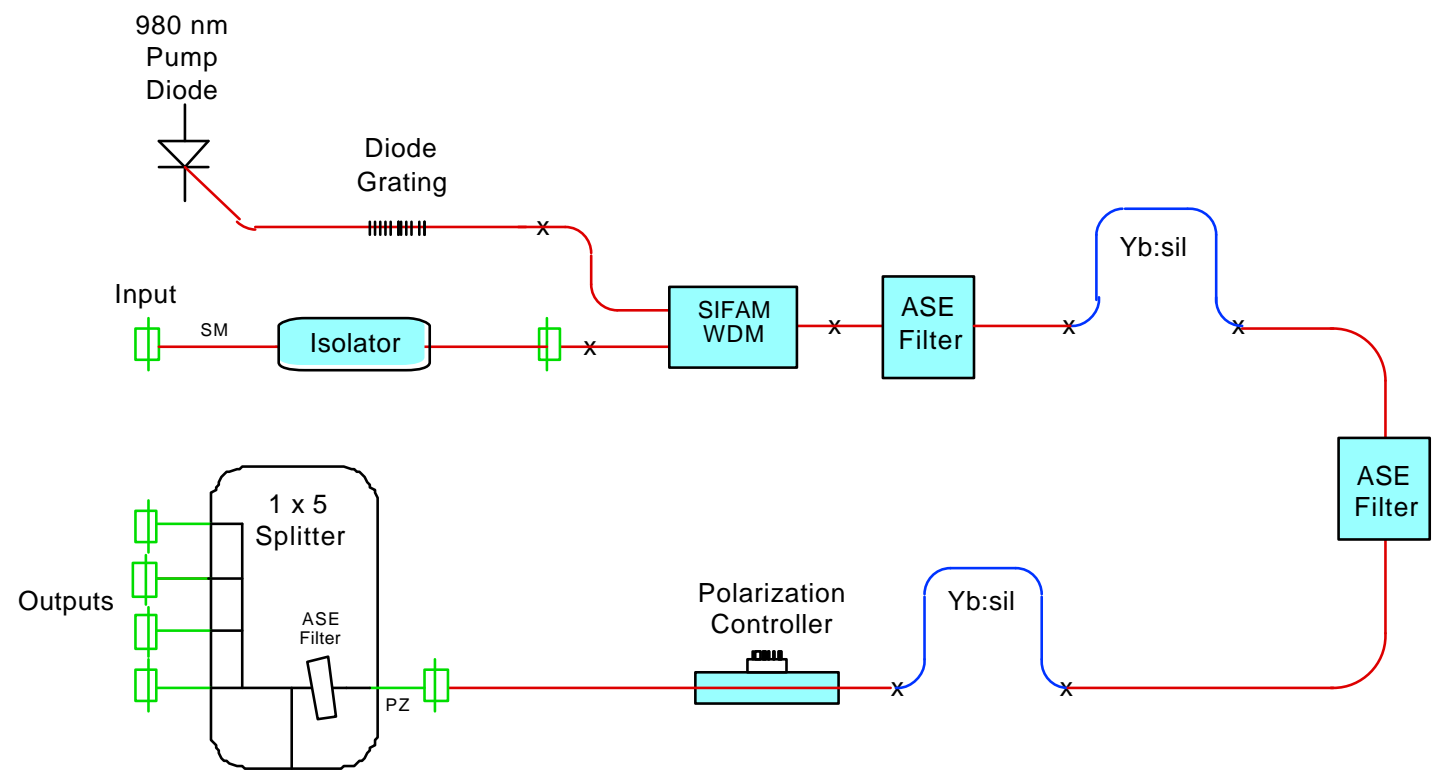

Figure 3. Optical schematic of fiber amplifiers used in the MOR.

\subsection{Phase Modulation System}

Phase modulation is applied to the single frequency light in the MOR for two different purposes. First, frequency modulation with a bandwidth of 1 angstrom at a modulation frequency of $3 \mathrm{GHz}$ is required to suppress buildup of SBS in the large aperture laser optics as well as the integrated fiber optics. Second, a bandwidth of 3-5 angstroms at $17 \mathrm{GHz}$ modulation frequency is required for smoothing of the speckle pattern illuminating the target (Smoothing by Spectral Dispersion [SSD]).

Both modulations are applied with an electro-optic waveguide modulator similar to those used in fiber-optic communications. The custom waveguide modulator design consists of 3 modulators on a single Lithium Niobate crystal. The first modulator is used at $3 \mathrm{GHz}$ for SBS suppression while the second two modulators are used to generate the required bandwidth for SSD. The waveguide modulators use only a few watts of RF power to produce the required bandwidth reducing the complexity of the drive circuit and enhancing the reliability.

\subsection{Optical Gate}

To reduce gain saturation in the fiber amplifiers and limit SBS effects in the fiber based system, the $100 \mathrm{~ns}$ pulse is reduced in a waveguide modulator to $45 \mathrm{~ns}$ before being introduced into the fiber amplifier and splitter array. The amplitude modulator design consists of two Mach-Zehnder interferometers driven by separate low voltage pulse generators. In addition to chopping out a $45 \mathrm{~ns}$ pulse, a modest temporal shape is added to compensate for the gain saturation in the fiber amplifier array. The modulator is a zero-bias design that maintains a minimum extinction of greater than $20 \mathrm{~dB}$ per modulator with no bias voltage applied. The modulator is a key element in the fail-safe design and acts as a fast shutter to inhibit the laser pulse if insufficient bandwidth is measured.

\subsection{Failsafe System}

The Failsafe System monitors the $3 \mathrm{GHz}$ modulation bandwidth of every pulse generated in the MOR at a $960 \mathrm{~Hz}$ rate. The $3 \mathrm{GHz}$ modulation is required to suppress buildup of SBS in the large aperture laser optics and MOR fiber optics. A pulse without sufficient bandwidth could potentially damage up to $2 / 3$ of the 192 beam lines of the NIF laser system.

The Failsafe System first converts the frequency modulation to amplitude modulation (FM-to-AM) by using a fiber 
grating. The grating is tuned so that the center frequency of the MOR light coincides with the edge of the grating transmission curve. The comb ination of the frequency modulation and the grating transmission curve generates amplitude modulation at the $3 \mathrm{GHz}$ phase modulation frequency. The magnitude of the amplitude modulation is directly proportional to the modulation bandwidth. The amplitude-modulated signal is filtered and converted to a simple pulse with an RF detector. The pulse amplitude is proportional to the bandwidth and is monitored by the Failsafe System. If the modulation falls below a predetermined set point, the Failsafe chassis inhibits the trigger to the optical gate chassis (described above) preventing the pulse from leaving the MOR. The optical delay between the Phase Modulator and Optical Gate chassis ( 500ns) provides sufficient time for the Failsafe System to measure and respond to every pulse generated in the MOR.

In addition to the bandwidth, the Failsafe System also monitors several other parameters including spurious AM, RF power, optical power and timing. Timing signals generated within the Failsafe chassis are used to determine if the phase modulation is present during the entire pulse. If any of the parameters are out of range, the Failsafe system inhibits the output of the laser pulse. Because of the importance of the Failsafe System, two redundant systems are used for each wavelength to increase the reliability. The Failsafe system is a critical component of the NIF laser system and has been rigorously tested to meet the NIF reliability requirements.

\subsection{FM-to-AM Compensation}

Phase (or frequency) modulation to amplitude modulation (FM-to-AM) conversion can be instigated by a large number of mechanisms within the NIF laser system. The common basis for all of these effects is non-uniform spectral transmission of either amplitude or phase. The MOR is tasked to pre-compensate for two such effects. First, the AM compensator, consisting of a tunable filter, is used to compensate for FM-to-AM generated by nonuniform spectral gain of the NIF amplifiers as well as non-uniform spectral transmission of optical elements. Second, the dispersion compensator, consisting of a grating stretcher, is used to compensate for the dispersion in the long fibers between the MOR and the NIF main amplifier system. Compensating for the AM reduces the peak power on NIF optics which increases the reliability of the system and allows for higher operating energies.

\subsection{Arbitrary Waveform Generator}

An array of fiber splitters and custom fiber amplifiers (described above) are used to provide 48 outputs with energies up to $200 \mathrm{~nJ}$ each. The final temporal pulse shape at the output of the MOR is sculpted from the $45 \mathrm{~ns}$ square pulse using Arbitrary Waveform Generators $(\mathrm{AWG})^{11}$. The AWG utilizes a 2-stage lithium niobate electro-optic modulator to vary the intensity of light in response to an electrical input. The electrical drive signal to the modulator is produced by a unique, high-performance AWG. The AWG sums the output of 140 electrical impulse generators each having $300 \mathrm{ps}$ pulsewidths and separated in time by $250 \mathrm{ps}$. By adjusting the amplitudes and summing the 140 pulses, an arbitrary electrical pulse shape can be generated. The second stage of the phase modulator is driven with a square pulse generator which is used to regulate the output energy. The automated pulse shaping system has demonstrated high contrast pulses (greater than 275:1) and is capable of matching the target wave shape within \pm $3 \%$ absolute when averaged over any 2 ns interval.

\subsection{MOR Summary}

The Master Oscillator Room of the National Ignition facility is an all fiber based laser system with proven long term stability. A subset of the final MOR system providing 4 output beams was first commissioned over five years ago.

This system has been operating continuously for over 40,000 hours with high availability and has met all critical NIF requirements. The system is currently providing 24 output beams and will be expanded to the final 48 output beams over the next year. 


\section{PRE-AMPLIFIER MODULE (PAM)}

\subsection{Regenerative Amplifier}

The NIF PAM Regenerative Amplifier (Regen) cavity consists of a diode pumped Nd:Glass rod (LG770), and a folded $665 \mathrm{~cm}$ ( $45 \mathrm{~ns}$ round trip) cavity. The $5 \mathrm{~mm}$ diameter amplifier rod is pumped at $800 \mathrm{~nm}$ by a $4 \mathrm{X} 1 \mathrm{~kW}$ diode array. Pump light is concentrated into the rod via use of a cored lens and hollow duct. The arrangement allows for a diffuse, end-pumped configuration while maintaining passage of the $1053 \mathrm{~nm}$ cavity beam. The pumped end of the rod is antireflection-coated at both wavelengths while the other end is coated for low reflection at $1053 \mathrm{~nm}$ and high reflection at $800 \mathrm{~nm}$. This amplifier head geometry enables positioning of the rod at a dynamically stable location, where the mode size is insensitive to surface figure perturbations on the rod facets and thermal lensing in the bulk. Figure 4 displays a schematic of the unfolded cavity.
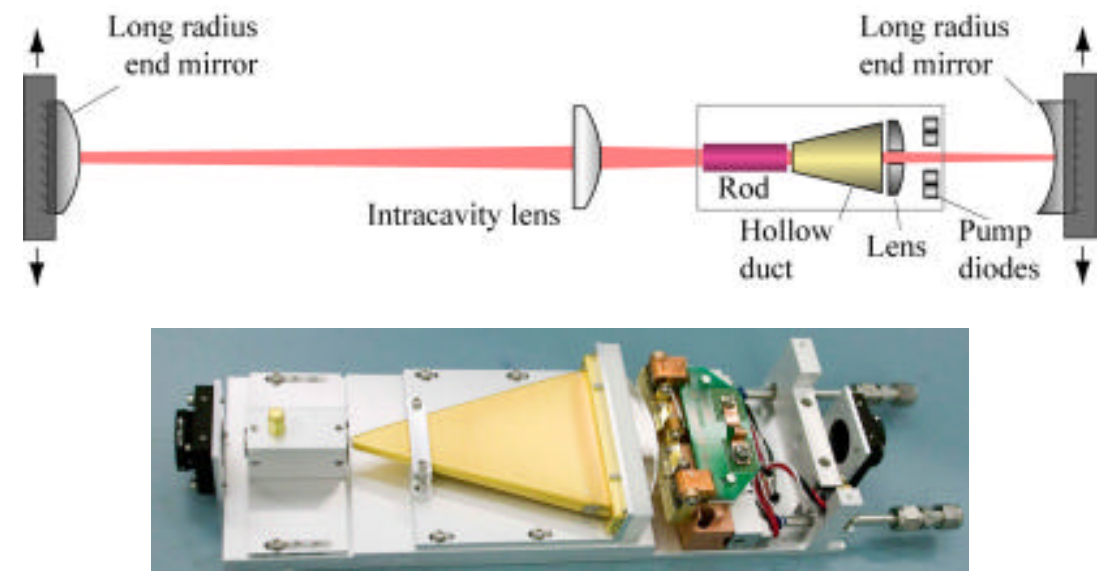

Figure 4. a) Unfolded regenerative amplifier cavity. A hollow lens duct endfire pump geometry is employed to concentrate $800 \mathrm{~nm}$ pump light from four $1 \mathrm{~kW}$ diode arrays into a $5 \mathrm{~mm}$ diameter, $5 \mathrm{~cm}$ long rod. Translation-mounted end mirrors possessing a long radius of curvature are employed for precise alignment. b) Photograph of the hollow lens duct amplifier head.

The design of the resonator cavity was developed by utilizing an analytic ABCD description of a simplified linear resonator cavity consisting of two end mirrors, a rod and an intra-cavity lens. Over 10,000 cavity configurations obtained by varying the end mirror and cavity lens radius of curvature and by changing the distance between the cavity elements (subject to several constraints - see below) were evaluated with respect to three different cavity design figure of merits (FOMs). Analytic expressions for the FOMs are based on a published theoretical description $^{1}$ of multi-element stable resonators. Due to the long cavity length, even weak focal lensing due to rod pumping and surface figure error can contribute to large changes in mode radius for non-optimized cavity configurations. We therefore treated the rod (in an $\mathrm{ABCD}$ sense) as a dielectric block (1/2 length of the rod), a thin lens with variable focal length $f$, and a second dielectric block ( $1 / 2$ length of the rod). The ABCD matrix for the cavity can then be written as

$$
\left.\left(\begin{array}{ll}
\mathrm{A}_{1} \mathrm{~B}_{1} \\
\mathrm{C}_{1} \mathrm{D}_{1}
\end{array}\right) \begin{array}{cc}
1 & 0 \\
-1 / f & 1
\end{array}\right)\left(\begin{array}{ll}
\mathrm{A}_{2} & \mathrm{~B}_{2} \\
\mathrm{C}_{2} & \mathrm{D}_{2}
\end{array}\right)=\left(\begin{array}{l}
\mathrm{D}_{1} \mathrm{~B}_{2}[n+\mathrm{u}] \mathrm{B}_{1} \mathrm{~B}_{2}[n+\mathrm{v}] \\
\mathrm{D}_{1} \mathrm{D}_{2}[n-\mathrm{v}] \mathrm{B}_{1} \mathrm{D}_{2}[n-\mathrm{u}]
\end{array}\right)
$$

where $A B C D_{1}$ is the matrix representing the cavity on one side of the rod, including the half of the rod and the end mirror curvature. Similarly $\mathrm{ABCD}_{2}$ represents the other half of the cavity, including an intra-cavity lens and end mirror curvature. The values $\eta, v$ and $u$ are functions of the $A B C D_{1,2}$ matrix elements:

$$
\begin{gathered}
n=\frac{1}{f}-\frac{1}{2}\left(\frac{\mathrm{A}_{1}}{\mathrm{~B}_{1}}+\frac{\mathrm{C}_{1}}{\mathrm{D}_{1}}+\frac{\mathrm{A}_{2}}{\mathrm{~B}_{2}}+\frac{\mathrm{C}_{2}}{\mathrm{D}_{2}}\right) \\
\mathrm{u}=\frac{1}{2 \mathrm{~B}_{1} \mathrm{D}_{1}}-\frac{1}{2 \mathrm{~B}_{2} \mathrm{D}_{2}}
\end{gathered}
$$




$$
v=-\frac{1}{2 B_{1} D_{1}}-\frac{1}{2 B_{2} D_{2}}
$$

The three FOMs used to develop an optimized resonator cavity for the NIF PAM Regen are:

FOM $_{1}$ : The cavity is stable over a large range of rod focal powers.

Due to the relatively long cavity length, small changes in the rod focal (due to thermal lensing or more importantly manufacturing tolerance) can lead to large variations in mode size (leading to undesirable disparities across the system of 48 PAMs) or unstable operation for many cavity designs. As a function of rod focal power, there are always two stability zones with identical width given by ${ }^{8}$

$$
\Delta \frac{1}{f}=\min \left(\left|\frac{1}{\mathrm{~B}_{1} \mathrm{D}_{1}}\right|\left|\frac{1}{\mathrm{~B}_{2} \mathrm{D}_{2}}\right|\right) .
$$

Only resonator designs stable over a range of $\Delta 1 / f= \pm 0.001 \mathrm{~cm}^{-1}$ centered at $1 / f=0$ were considered.

$\mathrm{FOM}_{2}$ : The cavity is dynamically stable.

This is equivalent to requiring the change in mode size in the rod $\omega_{\text {rod }}$ due to changes in the rod focal length be zero. We found that for some cavity designs, small changes in rod focal power changed $\omega_{\text {rod }}$ by several percent, in turn changing the output energy by $2 \Delta \omega_{\text {rod }} / \omega_{\text {rod. }}$. An analytic expression for the mode size in the rod for a dynamically stable cavity is given by ${ }^{12}$

$$
\omega_{\text {rod }}^{2}=\left(\frac{2 \lambda}{\pi}\right) \frac{1}{\Delta \frac{1}{f}}
$$

Again, only resonator designs meeting these criteria were considered.

$\mathrm{FOM}_{3}$ : The cavity misalignment sensitivity is minimized.

Again, because of the long cavity length, small drifts in end mirror alignment (or in the case of curved end mirrors, lateral displacement) can lead to large changes in mode placement in the rod, affecting energy stability and energy output. Additionally, changes in Regen pointing lead to centering changes on the beam shaping mask (pointing changes after the $20 \mathrm{X}$ beam expanding telescope are negligible). The displacement $\mathrm{x}_{\text {rod }}$ and propagation slope $\theta_{\text {rod }}$ in the rod due to end mirror tilt angles $\theta_{1,2}$ is given by the analytical expression ${ }^{1}$

$$
\left(\begin{array}{l}
x_{\text {rod }} \\
\theta_{\text {rod }}
\end{array}\right)=-\frac{1}{C}\left(\begin{array}{l}
\mathrm{D}_{2} \theta_{1}+\mathrm{D}_{2} \theta_{2} \\
-\mathrm{C}_{2} \theta_{1}+\left(\mathrm{C}_{1}-\mathrm{D}_{1} / f\right) \theta_{2}
\end{array}\right)
$$

where $C=-D_{1} D_{2}(1 / f+\xi-v)$. The optimal cavity design was based on cavities that met $\mathrm{FOM}_{1}$ and $\mathrm{FOM}_{2}$, minimized $1 / \mathrm{C}$ and met the various cavity design constraints listed below.

The design for the NIF PAM Regen was obtained by calculating the ABCD elements for over 10,000 cavity configurations obtained by changing: the end mirror focal lengths to very from $\pm 10 \mathrm{~m}$ including flat, the intra-cavity lens focal length to vary $\pm 10 \mathrm{~m}$ including no lens, the distance between mirror 1 and the rod, the distance between rod and intra-cavity lens and the distance between the intra-cavity lens and end mirror 2. In addition, only designs meeting the following constraints were analyzed: the resonator cavity length be $665 \mathrm{~cm}$ long, the mode radius in the rod be $1.5 \mathrm{~mm}$ (optimal mode size for a $5 \mathrm{~mm}$ diameter rod), the mode radius at end mirror 2 be $>0.9$ (to avoid damaging the Pockels cell located in front of end mirror 2, and the mode radius at end mirror 1 be $>0.6 \mathrm{~mm}$ (to avoid damaging the end mirror). 


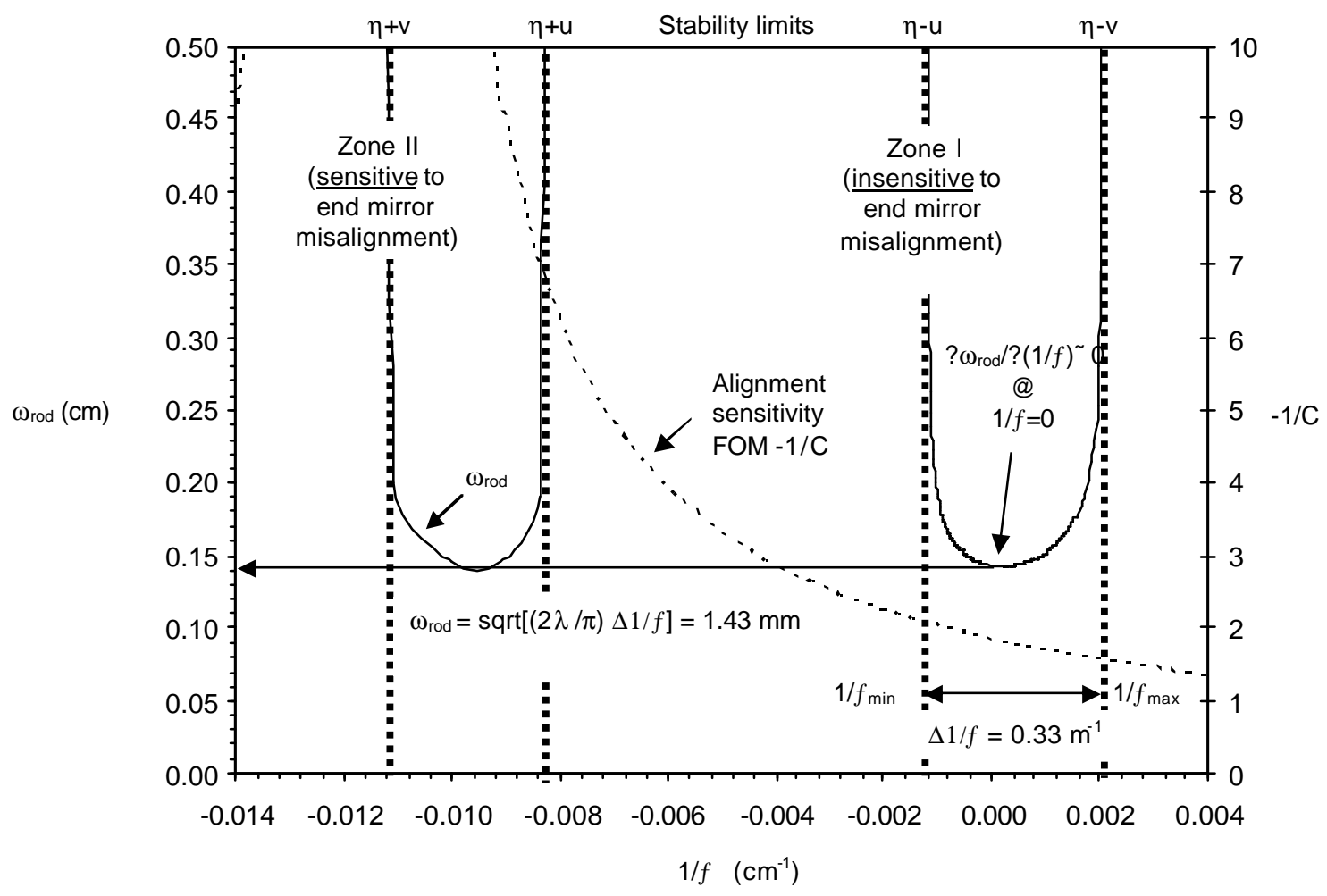

Figure 5. Plot of the rod mode radius $\omega_{\text {rod }}$ (solid line) for the two stability zones and misalignment FOM 1/C (dotted line) NIF PAM Regen design.

Figure 6 displays a photograph of the Regen cavity along with a schematic identifying key components. A Pockels cell in the cavity is used to Q-switch the MOR pulse into and out of the $45 \mathrm{~ns}$ cavity. Amplification takes place over 116 passes through the rod with a single-pass net gain factor of $\sim 1.21$. Energy saturation is achieved at $\sim 25 \mathrm{~mJ}$. The cavity mode size diameter is $2 \omega_{0}=3.1 \mathrm{~mm}$ in the rod. The cavity is aligned with long radius end mirrors mounted on translation stages for highly precise alignment and insensitivity to drift \& backlash. Measured mode profiles both imaging the rod and at the Regen are displayed in Figure 7.
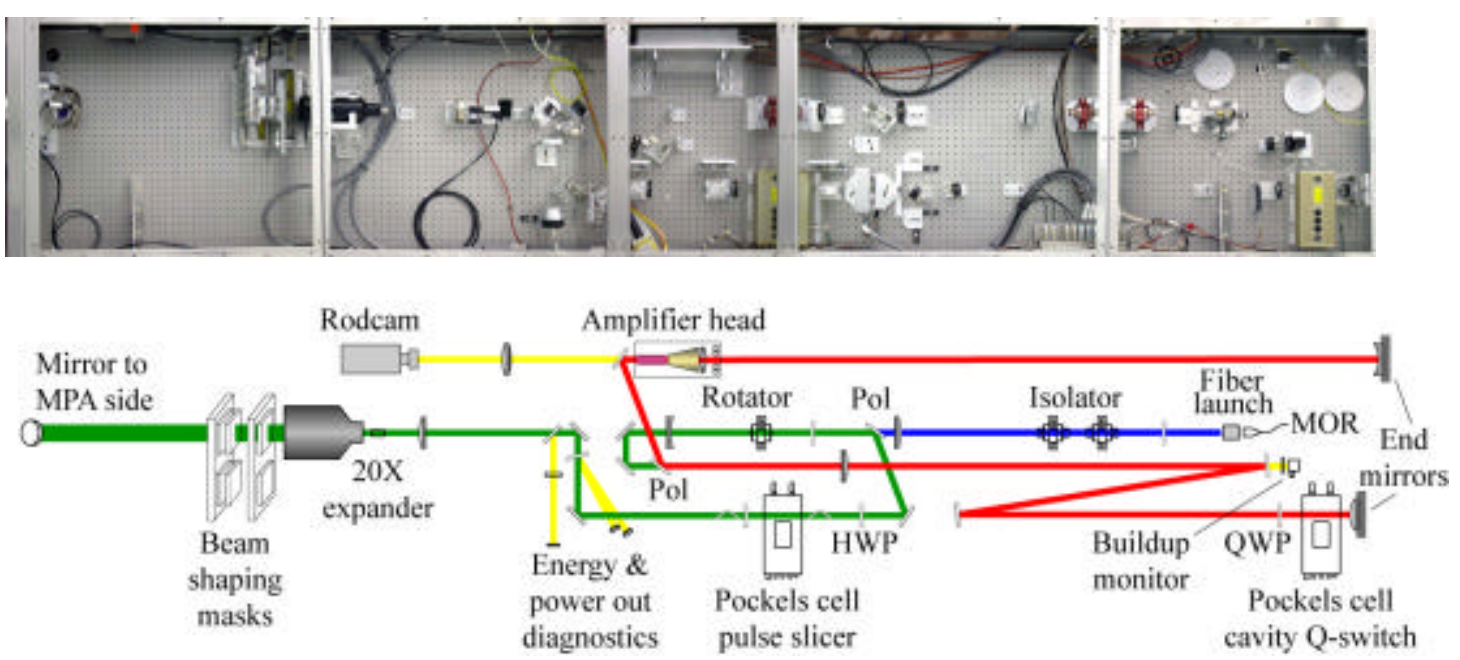

Figure 6 Regenerative Amplifier and Beam Shaping Module layout. The Regenerative amplifier cavity is seeded from the Master Oscillator Room (MOR), Q-switched / cavity-dumped in the resonant cavity, and extracted through a Faraday rotator. The output passes through a pulse slicer and is spatially formatted for injection into the Multi-Pass Amplifier (MPA). 

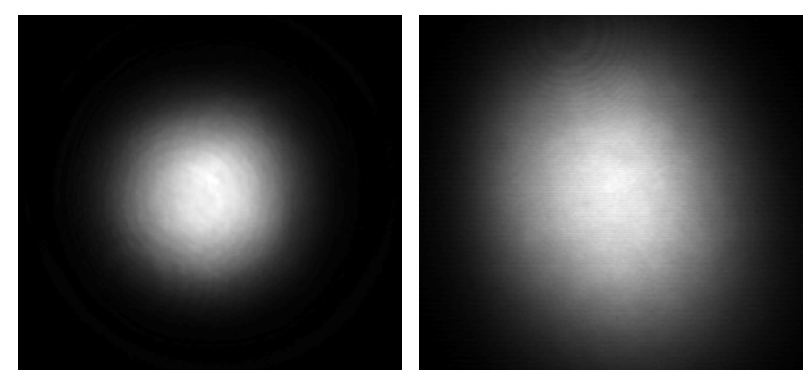

Figure 7 Regenerative Amplifier mode images. a) Image taken of the mode profile in the amplifier rod b) Image taken of the mode profile at the cavity output.

The output pulse passes through a Pockels cell slicer to cleanup residual prepulse power up to an extinction level of $>60 \mathrm{~dB}$. The output Gaussian beam is expanded up to $2 \omega_{0}=60 \mathrm{~mm}$ maximum and shaped into a apodized square profile with a serrated aperture apodizer mask ${ }^{13}$. The spatially-filtered serrated edge yields an apodized supergaussian edge profile. A shaping mask is optionally introduced to compensate for nonuniform spatial gain profiles both in the MPA and in the NIF main amplifiers. Fiducials are also optionally introduced in order to precisely center the beam output in the ISP. The shaped beam is then passed through a hole in the vertical breadboard onto the second stage of the PAM.

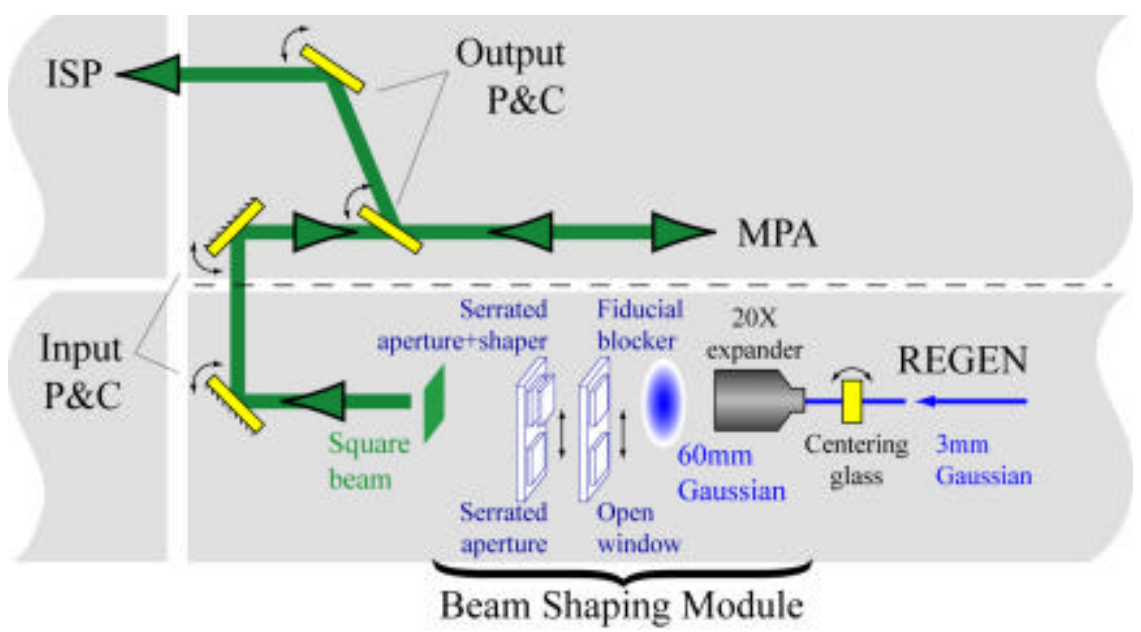

Figure 8 Control points used to align the beam through the Beam Shaping Module, into the MPA and out of the MPA into the ISP. The dashed line represents an unfolding of the breadboards that support the Regenerative Amplifier and MPA. A tiltable glass element is used to center the expanded regen Gaussian beam onto the shaping masks. Pairs of mirrors and polarizers are used to point \& center the beam into and out of the MPA.

The beam is injected through a pair of pointing and centering mirrors and a Faraday rotator before entry into the Multi-Pass Amplifier (MPA). Figure 9 displays a photograph of the MPA along with a schematic identifying key components. The MPA consists of a 4pass arrangement through a flashlamp-pumped $32 \mathrm{~mm}$ dia meter by $300 \mathrm{~mm}$ long rod. The ends of the rod are tapered to stifle unwanted whispering gallery parasitic modes that rob energy and burn o-ring seals. The flashlamp design incorporates 1.5" thick glass walls for increased mechanical durability. This beam formatting and subsequent losses through the MPA result in approximately $10 \%$ transmission. The voltage supplied to the flashlamps controls the gain which is adjusted to a single-pass value of $\sim 10$. The rod and flashlamps are sufficiently cooled after a 12 minute time interval for a subsequent shot. 

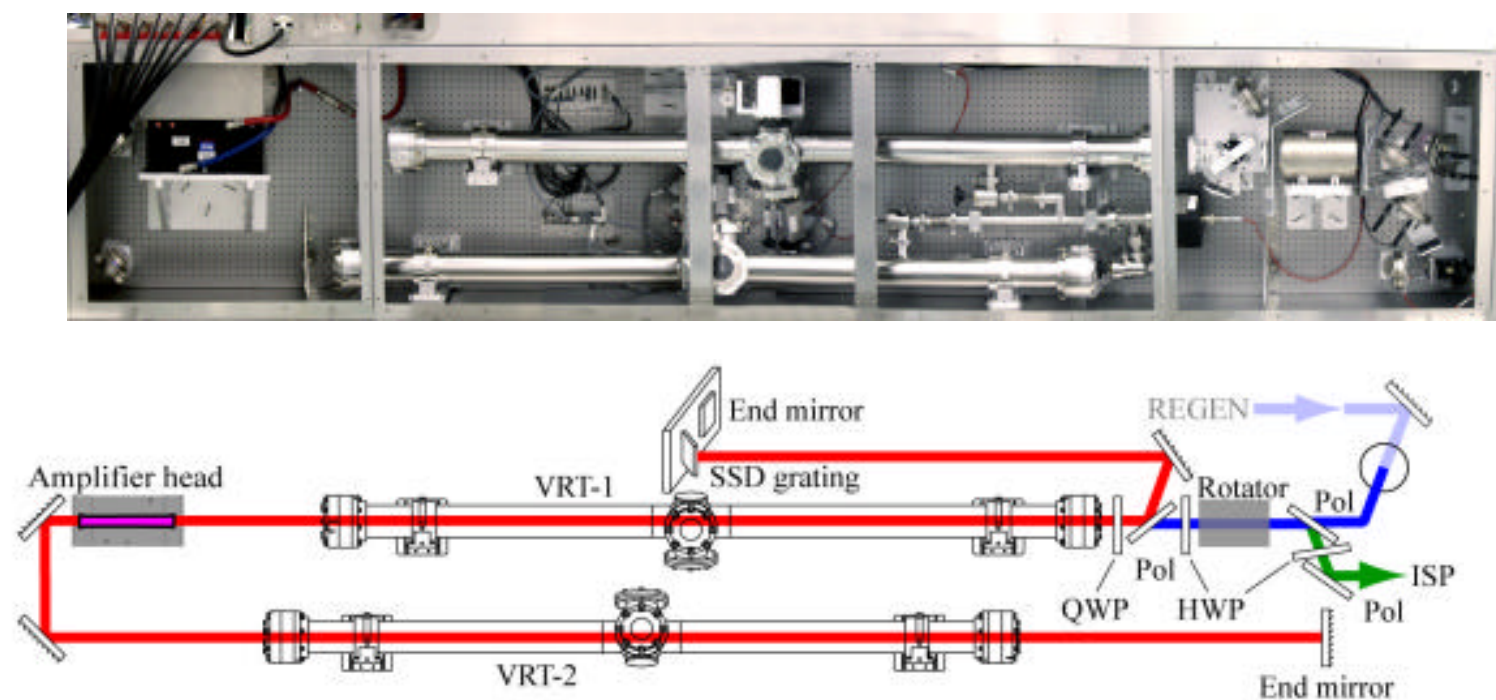

Figure 9. PAM Multi-Pass Amplifier (MPA) geometry. Following the Regenerative Amplifier stage and beam shaping module, the light is injected (blue) through a Faraday rotator into the four-pass amplifier circuit (red). The upper Vacuum Relay Telescope (VRT-1) images the shaped beam onto the flashlamp pumped rod. The lower Vacuum Relay Telescope (VRT-2) images the beam onto a displaced end mirror to avoid spatial hole burning and pulse shape distortion on the second pass. A QWP enables two more passes through the rod after reflecting off a choice of end mirror or SSD grating (at the Littrow angle) in the upper leg.

The square beam profile is spatially filtered and relay imaged through a pair of 2-element Vacuum Relay Telescopes (VRTs). The upper telescope (VRT -1) images the shaped beam onto the rod. The lower telescope (VRT -2) images the beam onto a displaced end mirror to avoid spatial hole burning and pulseshape distortion on the second pass. The third and fourth passes proceed after reflecting off of a choice of end mirror or SSD grating at the Littrow angle. The applied MOR phase modulation working in concert with the angular dispersion of the grating results in a smoothed farfield spot $^{14}$. Each VRT contains a 4-element pinhole array such that each of the 4-passes is angularly multiplexed. The lenses are further tilted to suppress unwanted reflections from entering the pinhole array and manifesting themselves as amplified pencil beams. The orientations of the tilts were strategically chosen to minimize net wavefront distortions well below the 1 wave peak-valley requirement. The system delivers nearly diffraction-limited performance as quantified in Figure 10. Further care has been taken to wedge and/or tilt remaining optical components and baffle mechanical components that give rise to stray reflections that result in parasitic oscillations ${ }^{15}$. The serrated aperture mask is implements a "blue chrome" process which includes both chrome and oxide layers for reduced reflectance. It is further tilted to reduce parasitic mechanisms resulting from reflections of the backside of the mask. 


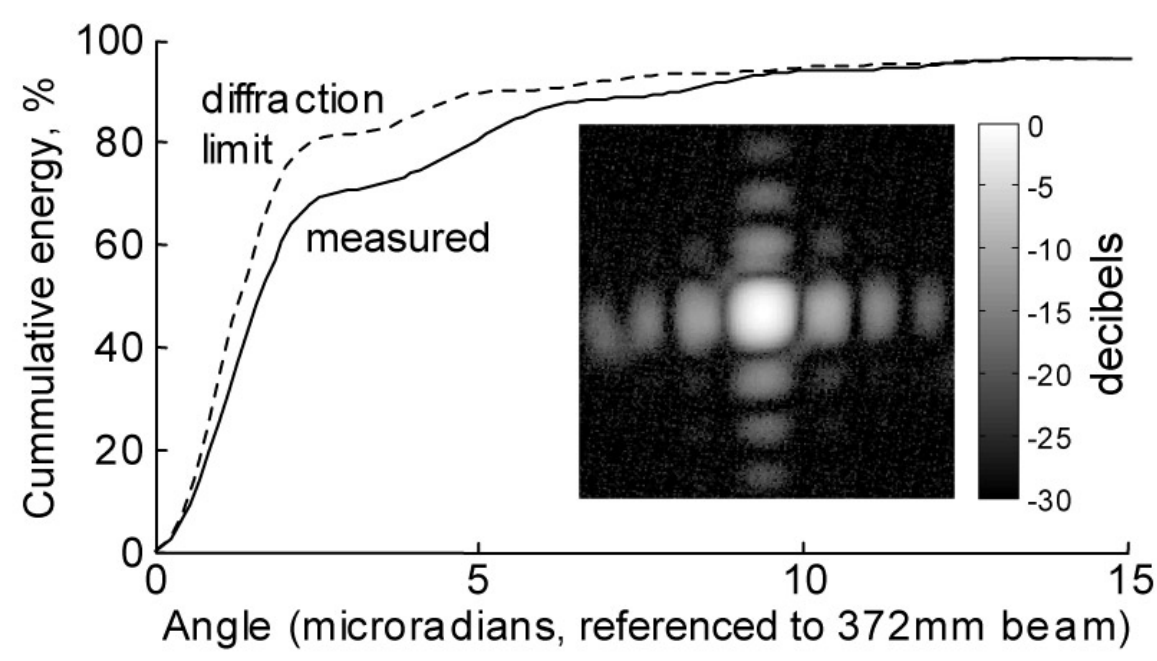

Figure 10. MPA output farfield cumulative angular energy with comparison to the diffraction limit for a perfect square beam. The PAM output beam is $18 \mathrm{~mm}$ square although the angular coordinate is downscaled to correspond to the angular space of the NIF main beam at $372 \mathrm{~mm}$ square. The inset displays the raw farfield spot data displayed on a logarithmic scale.

A quarter waveplate enables two more passes through the rod after reflecting off a choice of end mirror or grating (at the Littrow angle) in the upper leg. The $1050 \mathrm{lp} / \mathrm{mm}$ grating in conjunction with frequency modulation applied in the MOR results in a 1-D spatially-smoothed farfield spot.

After 4-passes and a net gain of $10^{4}$, the beam is amplified with a quadratic gain nonuniformity that is approximately 2.5:1 after 4passes for a $16 \mathrm{~mm} \times 16 \mathrm{~mm}$ square beam. The output beam passes through a pair of motorized polarizers that are used to clean up the polarization, attenuate the output in conjunction with a motorized halfwaveplate, and point and center the output beam. Figure 8 displays an overview of the control points used to align the input and output of the MPA.

The system of 48 PAMs are subject to a stringent set of requirements. A shot-to-shot energy stability requirement of $3 \%$ has been met for all PAMs. This is allocated in an RSS fashion as 1\% for Regen stability, $1 \%$ for spatial jitter at the mask, and $2.4 \%$ for the PAM gain. The requirement for nearfield contrast set at $5 \%$ has been met by all PAMs. A sample nearfield beam profile is displayed in Figure 11. Farfield pointing stability is kept below 1 microradian (RMS in reference to a $372 \mathrm{~mm}$ beam). 


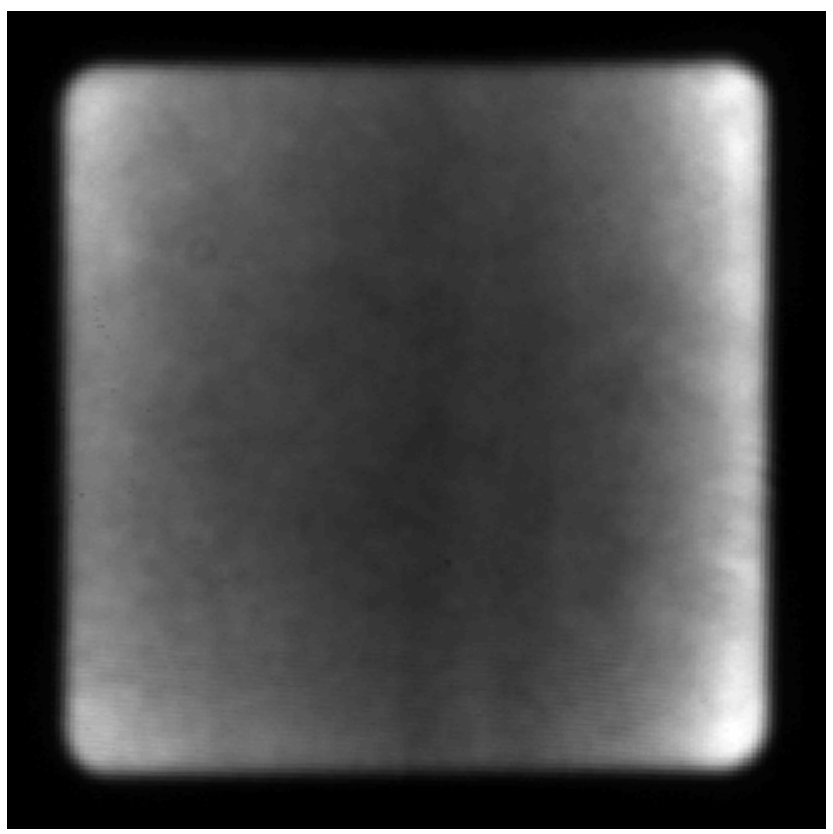

Figure 11. MPA output nearfield image at the Input Sensor Package. A high frequency contrast requirement of $5 \%$ is consistently met on all PAMs

All mounts on the PAM were carefully screened and pre-tested for optimum pointing stability. Accelerated lifetime tests were performed on motorized components expected to be frequently exercised over the projected 30 year NIF lifetime. These consist of the translation stages which toggle beam shaping and SSD grating options and rotation stages for half-waveplates used to adjust beam energetics. 


\section{THE INPUT SENSOR PACKAGE}

The Input Sensor Package (ISP) consists of a transport side and a diagnostic side on opposite sides of a 3' x 5' breadboard (see Figure 12). Each ISP serves four beamlines in the NIF, so there are 48 Input Sensor Packages in the National Ignition Facility. The transport side of an ISP includes a leaky mirror that transmits less than 5\% of the PAM energy toward the diagnostic side. The remaining laser light propagates from the PAM to the Pre Amplifier Beam Transport System via a second mirror, a Faraday isolator assembly, and an afocal vacuum relay telescope (VRT) that magnifies the beam and relays the pupil plane. These components include high fluence coated optics capable of handling up to $6 \mathrm{~J} / \mathrm{cm}^{2}$. The Faraday isolator assembly includes two polarizers, the Faraday rotator and a half-wave plate. The ISP transport side also contains an insertion mirror that sends the PAM beam to a calorimeter for energy calibration measurements, an insertion mirror to inject the $\mathrm{CW}$ laser from the diagnostic side (which is used for alignment and wavefront control for four NIF beam lines) and the Quad Back Reflection Sensor (QBRS). The QBRS consists of an energy diode in an integrating sphere that senses light returning from the four amplified beamlines. The Faraday isolator assembly prevents any undesired light reflected back from the main laser from reentering the PAM. This backward propagating linearly polarized light is instead transmitted back through the Faraday isolator and reflected off the polarizer into the QBRS diagnostic.

The $\mathrm{CW}$ alignment laser is located in the upper left quadrant of the diagnostic side and the beam is formatted into an $18.8 \mathrm{~mm}$ square beam with top-hat intensity profile prior to injection into the transport side of the ISP (see Figure 13). This laser is aligned into the Pre Amplifier Beam Transport System and is used as the alignment beam for each of four NIF beamlines. The ISP diagnostic side alignment path consists of a near field and far field component using a 12 bit digital camera. The near field and far field define the position and angular direction of the beam entering the PABTS so that the alignment into PABTS can be maintained or re-established using the ISP alignment diagnostic. This same alignment diagnostic is used to align the PAM beam into the PABTS on the same path as the $\mathrm{CW}$ alignment laser. Figure 14 shows an ISP installed in NIF, with the diagnostic side enclosures removed, adjacent to the PAM.

The CW laser can also be injected into a single mode fiber on the ISP which is then used as the wavefront reference and alignment reference by each of four NIF beams in another diagnostic system within NIF. The ISP diagnostic side also contains an Energy and Power measurement. Ninety percent of the light entering the diagnostic side is transmitted through a beam splitter into the Power measurement diagnostic. This diagnostic reduces the beam onto a phase plate and an integrating bar so that the power measurement is insensitive to spatial and angular variations in the measured beam. The laser light is routed via fiber to a diode and scope with a high gain and low gain channel in order to measure voltage versus time. The data from the two channels are stitched together to determine the temporal pulse shape. The low gain channel is too coarse to accurately measure the foot of the pulse, so a high gain channel takes this data prior to saturation from the peak of the pulse. Figure 15 is a plot of the voltage versus time stitched from the two channels from ISP Power sensor taken during a 1.8 MJ NIF campaign. The remaining 10\% laser energy is divided evenly between the energy diagnostic and the near field and far field alignment path. The Energy diagnostic contains a lens to focus $5 \%$ of the laser light onto a gold coated integrating sphere with a diode. Each of the diagnostic paths includes an optical input trigger which ensures all information from a system shot is properly captured. During a system shot, the diagnostic path is set to image the near field relay plane from the PAM. The spatial uniformity of the beam exiting the PAM can be evaluated from this image.

To ensure reliability of moving components over the 30 year NIF lifespan, lifetime cycle testing was performed. For example, the anticipated moves for the ISP CW laser insertion mirror is 200k moves, so this component was tested by our contract vendor, Adaptive Optics Associates, for 230k cycles. Initial testing showed that while the device did not have any functional issues, at $209 \mathrm{k}$ cycles, wear in the lead screw was causing rubbing and particulate generation. The assembly was subsequently re-designed and a modified assembly was retested successfully for 230k cycles 


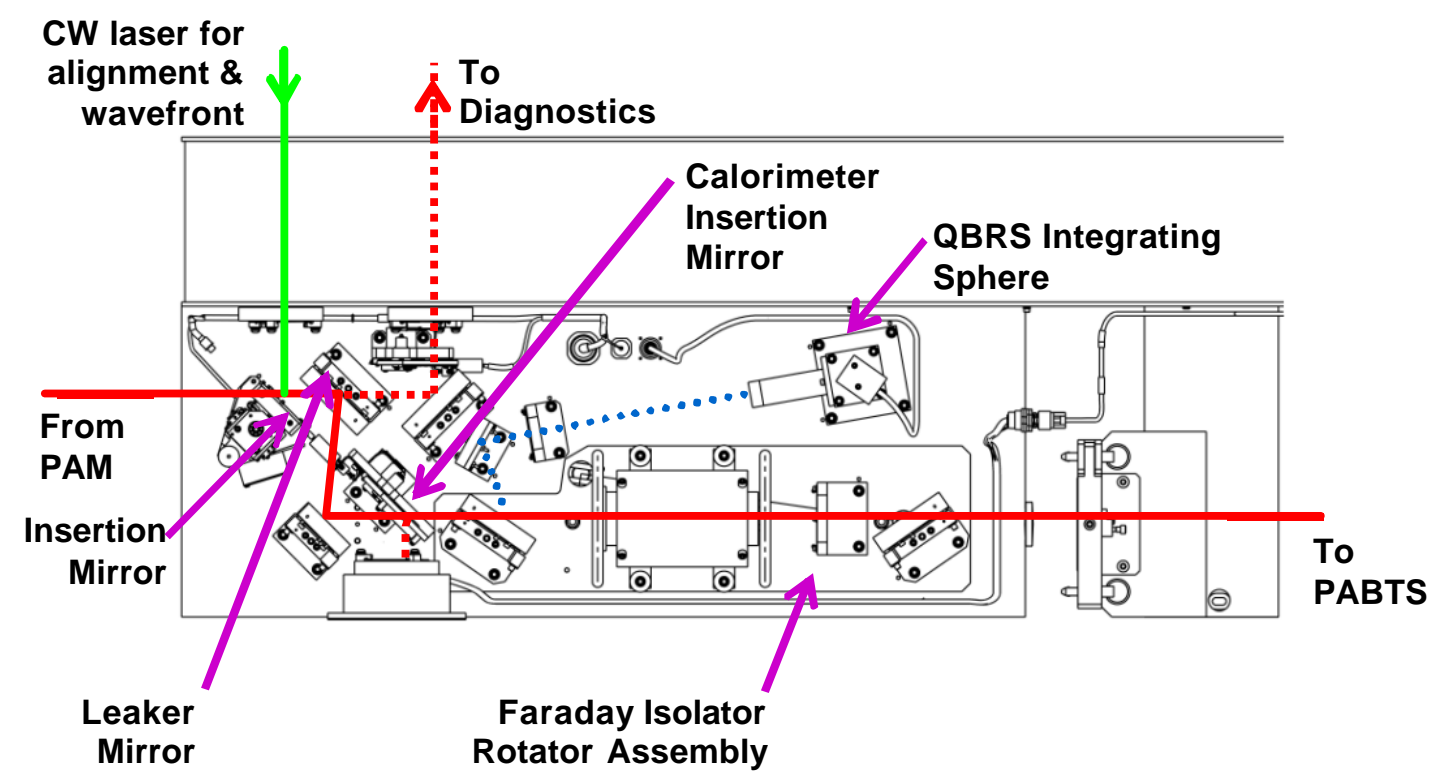

Figure 12. ISP Transport side showing laser propagation paths (VRT not shown)

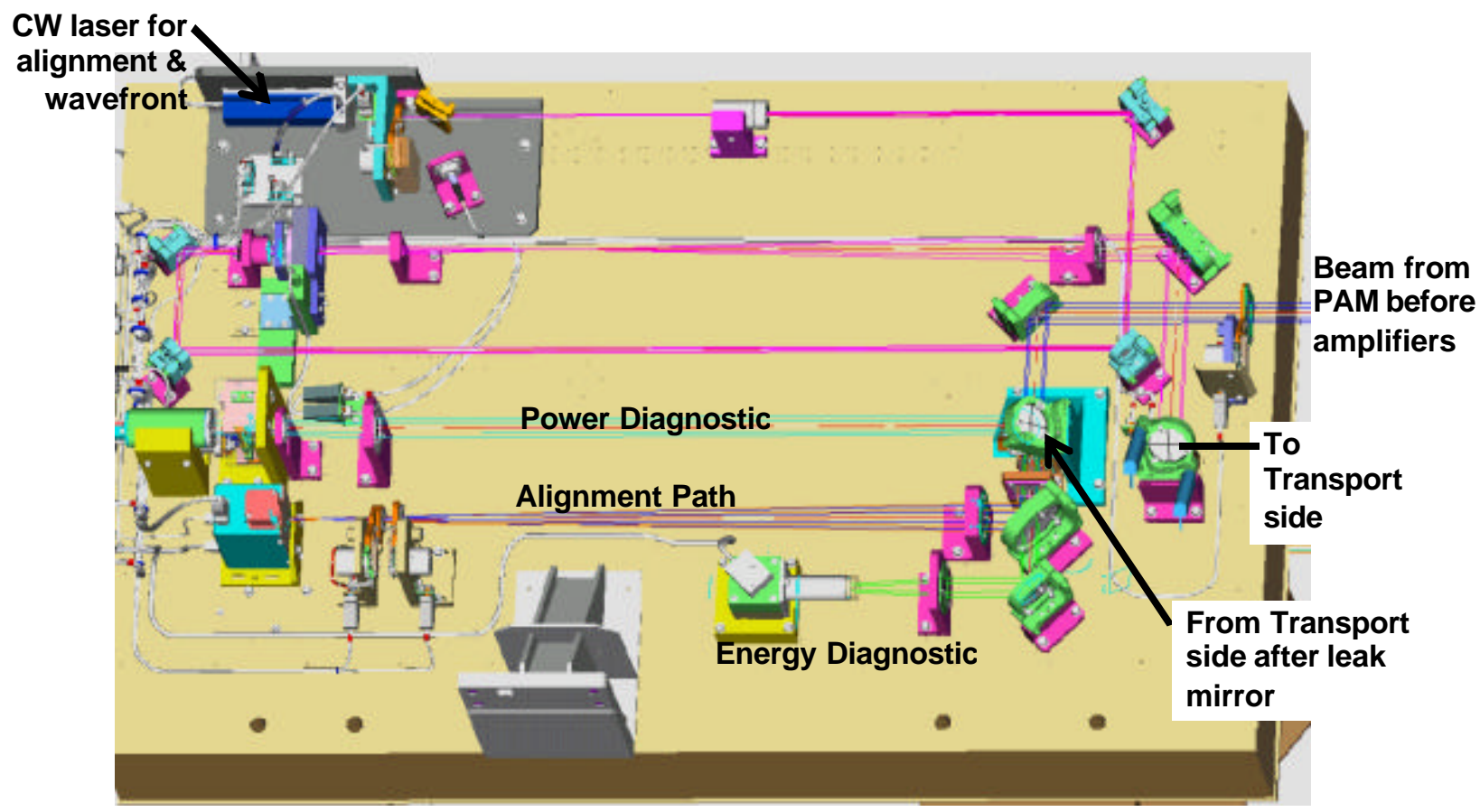

Figure 13. ISP diagnostic side showing laser propagation \& diagnostic paths 


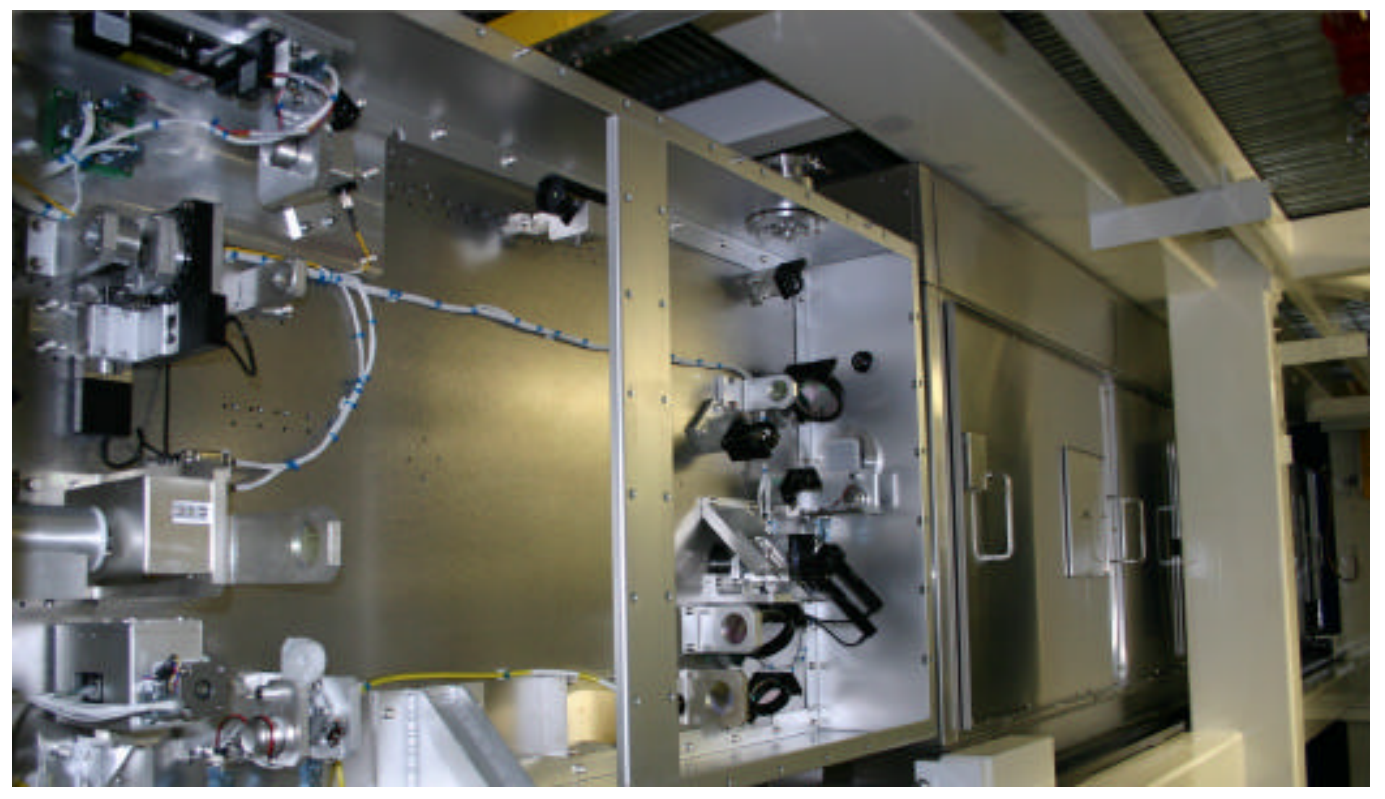

Figure 14. The ISP diagnostic side installed in NIF adjacent to an installed PAM

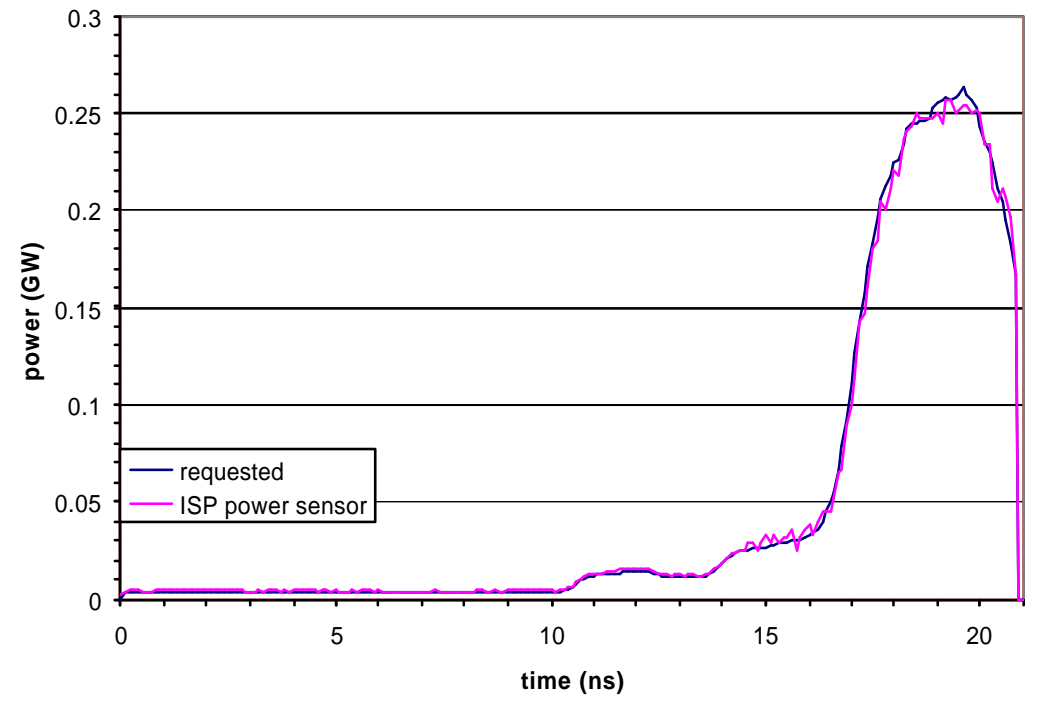

Figure 15. ISP Power Sensor pulse temporal data stitched from the high and low gain scope channels

\section{PABTS}

The Pre-A mplifier Beam Transport System (PABTS) consists primarily of mid-sized, discrete optics mounted on optical breadboards that split and format the PAM laser beams for injection into the Transport Spatial Filter (TSF). The design ${ }^{16}$ allows for individual beamline collimation, magnification, relay plane, and timing adjustment with an accuracy of better than $10 \mathrm{ps}$.

Like the PAM, the PABTS is mounted in a space frame called the Preamplifier Support Structure. Unlike the PAM, the PABTS consists of discrete optics individually installed in the field on optical breadboards. The tables are essentially standard optical breadboards with custom mounting holes as well as holes through which laser beams must pass in the process of being split and formatted. In order to minimize deflection, each of these breadboards is supported at four corners by spherical bearings mounted to the Preamplifier Support Structure. In addition to 
supporting the PAM and PABTS the structure also supports 100 tons of spatial filter vacuum vessels for the main beam transport optics.

The optical components of the PABTS include high-reflectors, polarizers, half-wave plates, and multiple-element vacuum relay telescopes. At this point in the NIF beam path, the energy is still low, on the order of a joule, and the beam can be on the order of $30 \mathrm{~mm}$ x $30 \mathrm{~mm}$ without exceeding damage fluence limitations. In keeping with the strategy of much of the Injection Laser System, we have adapted commercial mirror mounts of the three, four, and five inch varieties throughout the PABTS. This resulted in significant cost savings. All commercial mounts used in

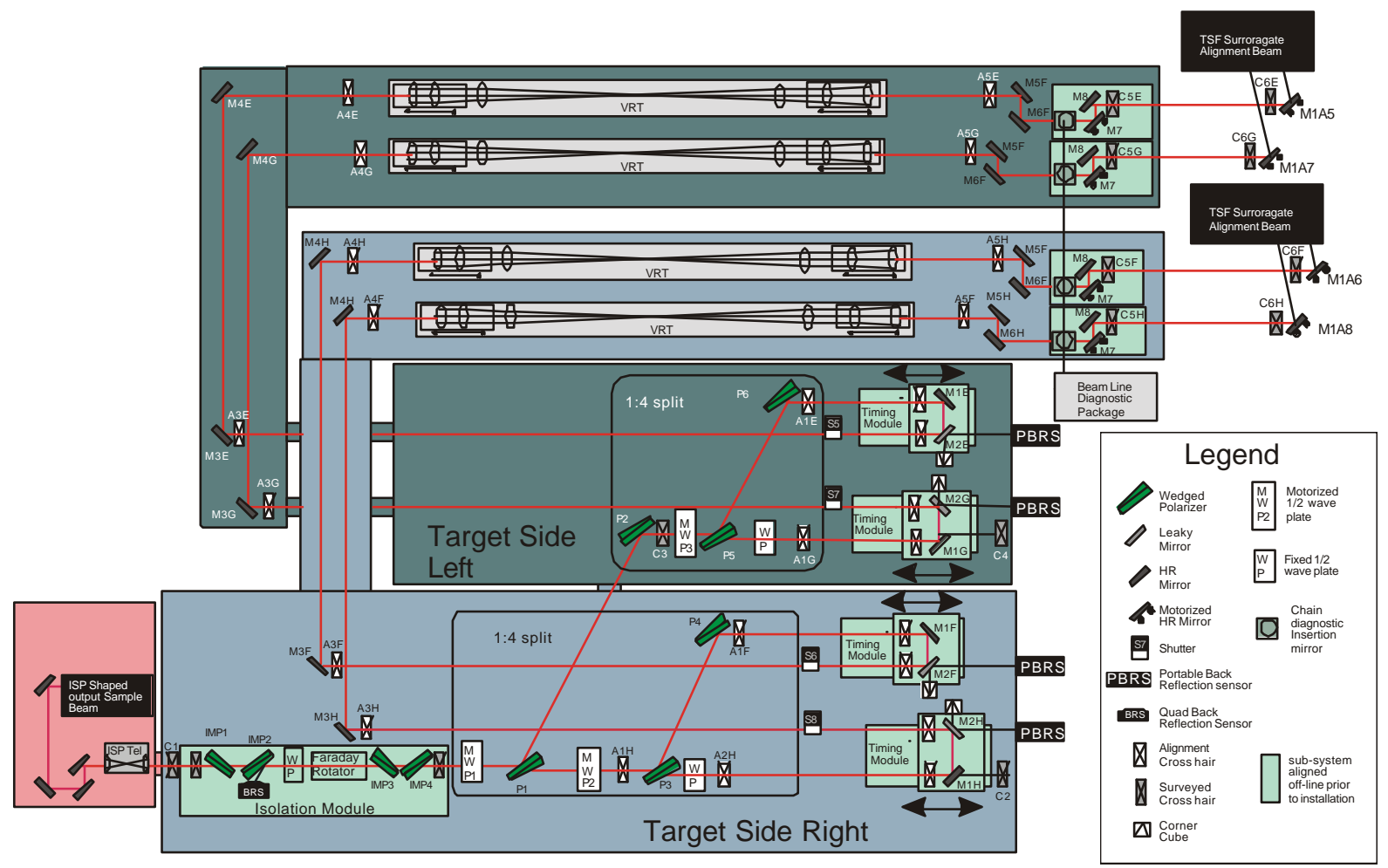

Figure 16. The Preamplifier Beam Transport system.

the ILS were tested for short and long term drift, wavefront distortion on the installed optic, and resistance to external shocks.

Enclosures cover the optical tables and enclose the optical components in what is effectively a Class 100 clean room. During operating conditions, a low flow of humidity controlled, HEPA filtered air is supplied to the enclosures to maintain cleanliness. Maintenance, however, is performed through $1 \mathrm{~m} \mathrm{x} 1 \mathrm{~m}$ openings after first pressurizing the enclosures with portable HEPA filters designed to provide a nominal 15 linear meters per minute of flow out of the enclosure opening.

The formatting of the laser beams prior to injection into the Transport Spatial Filter is accomplished for each beam with a four-element symmetric vacuum relay telescope (VRT). A VRT has a vacuum region between the inner two elements that prevents air breakdown at the focus. Collimation and size are adjusted by translating the outer elements. Changing the position of the telescope along the optical axis controls pupil relay. The design of the PABTS VRTs, like all of the ILS VRTs, uses fixed optical mounting with no tip-tilt adjustments. This significantly reduces fabrication, assembly, and maintenance costs. To maintain vacuum, the eight VRTs of a bundle are manifolded together and are pumped with a 70 liter per second turbo pump. 


\section{AUTOMATIC ALIGNMENT}

\subsection{Automatic Alignment Introduction}

NIF PAM alignment is performed automatically, utilizing four fixed references for centering and pointing, and an image template for beamshaper positioning. Input alignment is performed to position the beam within the PAM beampath at the MM4 (lower) end mirror and at the focal (pinhole) planes. Output alignment co-aligns the PAM beam to the same references used by the Input Sensor Package (ISP) cw beam. A centering glass provides precision positioning of the nominally Gaussian intensity profile Regen beam onto the Beam Shaping Mask (BSM).

Alignment proceeds in the sequence as follows:

1. Center the Regen beam onto the BSM to achieve the required beam shape. The centering glass adjusts the position of the RA Gaussian profile beam on the BSM until the profile matches a predetermined image template.

2. Center the beam to PAM MPA end mirror using the $980 \mathrm{~nm}$ source as a reference, by adjusting the input pointing and centering mirrors (Figure $8 \&$ 9).

3. Point the beam to the Vacuum Relay Telescope \#2 (VRT 2) pass 4 pinhole which is backlit by the same $980 \mathrm{~nm}$ source. This pointing uses the same input pointing and centering mirrors as in step 2.

4. Center the beam to the Input Sensor Centering Refe rence Mask, using the output pointing and centering polarizers (Figure $8 \& 9$ ).

5. Point the beam to the Input Sensor to a pre-determined camera pixel location using the output pointing and centering mirrors.

All these alignment steps presume the system is 'reasonably' aligned, and adjustments of no more than $30 \%$ or so of the CCD camera field of view are required, and that the Near Field view is not clipped (Fig. 18). Practice has shown that decentering of no more than a few pixels is required to realign the beam on a daily basis. Relative alignment of all the other PAM optics is fixed during assembly and factory commissioning, and on-line adjustment is required infrequently and only as a maintenance activity.

To accomplish each of these alignment operations, numerous devices and sensor settings are modified to image first the reference followed by the 'beam' which is being positioned relative to the reference, and in essence the CCD camera temporarily captures the reference position for the duration of that alignment operation (Fig 19). Shutters, attenuators, insertable lenses, and mirrors must be actuated. In addition, combinations of the four actuators for each mirror pair are actuated to independently center and point the beam. This is accomplished through a device matrix for each alignment pair and mirror pair, a table of values calculated from measurements during PAM commissioning.

With 48 PAMs to be aligned rapidly, in an error-free manner, the Automatic Alignment System requires reliable device operation, advanced image processing, and a sophisticated control system. Each of these components is described further as follows.

\subsection{Devices and Actuators}

Most NIF devices are stepper motors, such as all mirror actuators, and the majority of the devices within the PAM and Input Sensor package. Stepper motors are simple devices, but each has a detailed set of requirements for acceleration, speed, drive current and numerous other operational parameters. We have found during NIF early light experiments and initial NIF commissioning and operation that device reliability is paramount to successful automatic alignment. At the most basic level, each motor actuation to other than an endpoint (designated by a limit switch) utilizes the concept of 'backlash' to approach each designated motor stepcount from the positive direction. Since nearly all stepper motor positioners have a 'dead zone' when reversing direction, each motor is deliberately driven past the desired position by a number of motor stepcounts (defined as the 'backlash' value), and then driven back in the negative direction until the specific motor stepcount is again reached. During the commissioning of each motor, the backlash is measured and installed into the operational database using an automated computerized Maintenance and Commissioning Tool. Many other motor parameters are universal across NIF for a particular device, parameters determined in many cases from extensive measurement and analysis. The same process has been applied for limit switches, which are called upon to indicate end-of-travel for binary devices. Much investigation 
into switch behavior was performed, both to identify optimally suited switches, and to favorably implement switches already designed into the system.

\subsection{Image Processing}

A key element of reliable automated alignment is robust image processing. There are variations between the PAM's and ISP's including slight beam rotation, camera and optic defects, stray light, and other image flaws to which the image processing must adapt. But the image processing must also not 'find' beam fiducials for images which are in error such as improperly illuminated or even the wrong image due to actuator malfunction. Thus, we have defined a specific set of requirements of acceptable images for PAM alignment. The image processing strategy includes preprocessing algorithms to immediately reject bad images such as blank images or the wrong images due to actuator malfunction. Once an image is accepted for processing, an algorithm specific to the image is utilized to find the
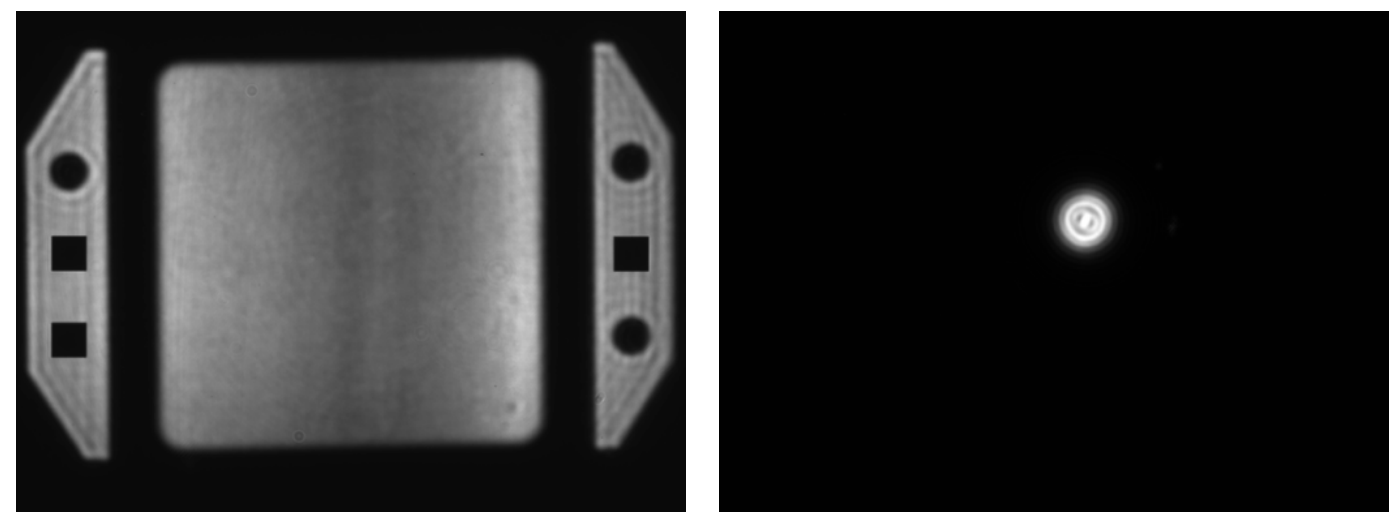

Figure 18. Image of the PAM nearfield beam and the mirror MM4 centering reference. The center region is propagated to the main laser, the 'wing' regions are used for beam centering alignment. The round 'fiducial' marks represent the PAM beam position, which are centered to the reference for input alignment using mirrors BM1/MM1. The beam is also centered to the square ISP fixed centering mask fiducials during PAM output alignment using polarizers POL1/POL3.

image fiducials and determine their position on the camera. This information is then passed back to the automatic alignment routine which compares beam to reference, and adjusts the beam position as required.

\subsection{Control System}

Rapid, fully automated alignment is accomplished on PC based computers, an image processing cluster (described above), and powered by software written in Java, Ada, and the commercial IDL package. The main software in Java and Ada permits an operator or higher-level control system to select a set of beams up to all 48 PAM's, and perform full alignment. The software sets up all the devices to acquire the reference and beam images in sequence, and commands the mirror pairs to close the loop based u pon the reference $(\mathrm{x}, \mathrm{y})$ - beam $(\mathrm{x}, \mathrm{y})$ error, in the loop order listed above. The loop repeats with diminishing gain until the error falls below a predefined value. Well behaved devices permit rapid loop closure, but in contrast, devices which fail to actuate properly force actuation retries and loop repeats. When completed, all the images and actuations are logged for diagnostic purposes. Large numbers of images are filed away, and the image processing scientists use libraries of hundreds to thousands of such images to exercise their algorithms.

\section{INTEGRATED INJECTION LASER SYSTEM}

The integrated ILS must maintain its reliability under a variety of pulse shapes, energies, durations, and modulation bandwidth conditions. In order to assure high reliability without damage to the laser system, a set of checks and balances has been employed. The MOR is constantly monitoring the pulse shape and energy. The monitoring occurs with approximately 100 pulse averages occurring at the $960 \mathrm{~Hz}$ MOR pulse repetition frequency. Thus the response to an incorrect pulse shape is not instantaneous, but rather on the order of $100 \mathrm{~ms}$. Slow degradation of any MOR pulse shape would not damage the other optics in the system due to additional checks described below. The 
only realistic way that damage could occur in the Regen would be during pulse shaping. The controlling software is used to verify that all Regen shutters are closed during pulse shaping operations. Once pulse shaping is complete, the active control of the FET's is stopped. The amplitude is adjusted with a separate control to the DC bias on the amplitude modulator. Once the Regen shutter is open, the output energy is actively monitored after the variable attenuator is used to set the NIF shot energy. If that energy is more than $2 \%$ outside of the required energy for the shot, the shot is aborted. This, combined with the MOR pulse shape monitoring, makes the system very reliable for rod shots (PAM MPA active, Main Amplifier dormant). After each rod shot, the energy, the pulse shape, and the near field profile are monitored at the input sensor package (ISP) and compared to the requested configuration by the Laser Performance Optical Model (LPOM). All critical devices are reserved (cannot be moved) before each rod shot. If the rod shot pulse shape at the ISP meets all laser performance criteria, the software will proceed on to the system shot (PAM MPA and Main Amplifier both active). With all critical devices reserved, the MOR pulse shape checks, and the Regen energy monitoring we guarantee with high confidence that the appropriate spatial pulse shape, temporal profile, and energy are obtained on the system shot.

\section{References}

1. J. A. Paisner, J. D. Boyes, S. A. Kumpan, W. H. Lowdermilk, and M. Sorem, "Conceptual Design of the National Ignition Facility," Proceedings of the First International Conference on Solid State Lasers for Application to Inertial Confinement Fusion, William F. Krupke, Editor, SPIE Proceedings 2633, pp. 2-12, Monterey, CA, 1995.

2. R. E. English, Jr., C. W. Laumann, J. L. Miller, and L. G. Seppala, "Optical system design of the National Ignition Facility," International Optical Design Conference, Leo R. Gardner, Kevin P. Thompson, Editors, SPIE Proceedings 3482, pp. 726-736, Kona, HI, CA, 1998.

3. J. K. Crane, R. B. Wilcox, N. W. Hopps, D. Browning, M. D. Martinez, B. Moran, F. Penko, J. E. Rothenberg, M. Henesian, C. B. Dane, and L. A. Hackel, "Integrated operations of the National Ignition Facility (NIF) optical pulse generation development system”, Third International Conference on Solid State Lasers for Application to Inertial Confinement Fusion, W. H. Lowdermilk; Editor, SPIE Proceedings 3492, pp. 100-111, Monterey, CA, 1999.

4. P. J. Wisoff, M. W. Bowers, G. V. Erbert, D. F. Browning, and D. R. Jedlovec, "NIF Injection Laser System," Optical Engineering at the Lawrence Livermore National Laboratory II: The National Ignition Facility, Monya A. Lane, Craig R. Wuest, Editors, SPIE Proceedings 5341, pp. 146-155, San Jose, CA, 2004.

5. J. K. Crane, M. D. Martinez, B. D. Moran, C. W. Laumann, J. M. Davin, R. J. Beach, B. P. Golick, R. L. Jones, J. Braucht, M. D. Perry, K. M. Skulina, F. A. Penko, S. M. Herman, S. C. Burkhart, and S. C. Mitchell, "Description and performance of the preamplifier for the National Ignition Facility (NIF) laser system," Solid State Lasers for Application to Inertial Confinement Fusion: Second Annual International Conference, Michel L. Andre, Editor, SPIE Proceedings 3047, pp. 601-609, Monterey, CA, 1997.

6. M. D. Martinez, K. M. Skulina, F. J. Deadrick, J. K. Crane, B. Moran, J. Braucht, B. Jones, S. Hawkins, R. Tilley, J. Crawford, D. Browning, and F. Penko, 'Performance results of the high gain, Nd:glass, engineering prototype preamplifier module (PAM) for the National Ignition Facility (NIF)", Laser Resonators II, Alexis V. Kudryashov, Editor, SPIE Proceedings 3611, pp. 169-180, San Jose, CA, 1999.

7. J. R. Murray, J. R. Smith, R. B. Ehrlich, D. T. Kyrazis, C. E. Thompson, T. L. Weiland, and R. B. Wilcox, "Experimental observation and suppression of transverse stimulated Brillouin scattering in large optical components,” J. Opt. Soc. Am. B. 6, pp. 2402-2411, 1989.

8. M. D. Martinez, J. Crane, F. Penko, and D. Browning, "Optimized, Diode-Pumped, Nd:Glass, Prototype Regenerative Amplifier for the National Ignition Facility," Optoelectronics and High-Power Lasers \& Applications, Alexis V. Kudryashov; Pierre Galarneau; Editors, SPIE Proceedings 3267, pp. 234-242, Monterey, CA, 1998.

9. N. W. Hopps, R. B. Wilcox, M. R. Hermann, M. D. Martinez, E. H. Padilla, and J. K. Crane, "Optimization of the Alignment Sensitivity and Energy Stability of the NIF Regenerative Amplifier," Third International Conference on 
Solid State Lasers for Application to Inertial Confinement Fusion, W. H. Lowdermilk; Ed itor, SPIE Proceedings 3492, pp. 991-996, Monterey, CA, 1999.

10. A. Asseh, H. Storoy, J.T. Kringlebotn, W. Margulis, B. Sahlgren, S. Sandgren, R. Stubbe, and G. Edwall, "10 cm Yb3+ DFB fibre laser with permanent phase shifted grating,” Electron. Lett. 31, pp. 969-970, 1995.

11. S. C. Burkhart, R. B. Wilcox, D. Browning, and F. Penko, "Amplitude and Phase Modulation with Waveguide Optics," Solid State Lasers for Application to Inertial Confinement Fusion: Second Annual International Conference, Michel L. Andre, Editor, SPIE Proceedings 3047, pp. 610-617, Monterey, CA, 1997.

12. V. Magni, "Multielement stable resonators containing a variable lens," J. Opt. Soc. Am. A., 4, pp. 1962-1969, 1987.

13. J. M. Auerbach and V. P. Karpenko, "Serrated-aperture apodizers for high-energy laser systems, “ Appl. Opt. 33, pp. 3179-3183, 1994.

14. S. Skupsky, R. W. Short, T. Kessler, R. S. Craxton, S. Letzring, and J. M. Soures, "Improved laser-beam uniformity using the angular dispersion of frequency-modulated light," J. Appl. Phys. 66, pp. 3456-3462, 1989.

15. B. D. Moran, C. B. Dane, J. K. Crane, M. D. Martinez, F. Penko, and L. A. Hackel, "Suppression of Parasitics and Pencil Beams in the High-Gain National Ignition Facility Multipass Preamplifier," Optoelectronics and HighPower Lasers \& Applications, Santanu Basu, Editor, SPIE Proceedings 3264, pp. 56-64, Monterey, CA, 1998.

16. C. Laumann, R. Korniski, "NIF optical systems design, preamplifier beam transport system opto-mechanical design," $44^{\text {th }}$ Annual Meeting of the International Symposium on Optical Science, Engineering, and Instrumentation, H. Philip Stahl, Editor, SPIE proceedings 3782, pp. 488-495, Denver, CO, 1999.

17. J. V. Candy, W. A. Mcclay III, A. S. Awwal, S. W. Ferguson, "Detection and Classification of off-normal Images During Automatic Alignment of High Energy Laser Beams,"

This work was performed under the auspices of the U. S. Department of Energy by University of California, Lawrence Livermore National Laboratory under contract W-7405-Eng-48. 